\title{
Comprehensive Transcriptome and Metabolome Analysis of Lithocarpus polystachyus Leaf Revealed Key Genes in Flavonoid Biosynthesis Pathways
}

\author{
Kai-Xiang Li \\ College of Biological Sciences and Technology, Beijing Forestry University, Beijing, 100083, China; \\ and Guangxi Forestry Research Institute, Nanning, 530002, China \\ Kai Liu \\ Guangxi Forestry Research Institute, Nanning, 530002, China \\ Yingying Chen \\ Guangxi Forestry Research Institute, Nanning, 530002, China; and College of Biological Sciences \\ and Technology, Beijing Forestry University, Beijing, 100083, China
}

Xiaolu Huang, Wenhui Liang, and Baocai Li

Guangxi Forestry Research Institute, Nanning, 530002, China

Yingbai Shen

College of Biological Sciences and Technology, Beijing Forestry University, Beijing, 100083, China

Haiying Liang
Department of Genetics and Biochemistry, Clemson University, Clemson, SC 29634

ADDITIONAL INDEX wORDS. differentially accumulated secondary compounds, differently expressed genes, RNA sequencing, sweet tea

\begin{abstract}
Lithocarpus polystachyus is a unique medicinal tree species that is valued for its abundant flavonoids in leaves. Currently, genes and metabolites involved in the flavonoid biosynthesis pathway remain largely unknown. To elucidate the flavonoid biosynthesis pathways, transcriptome and metabolome analyses of young, mature, and old leaves were conducted. A total of 86,927 unigenes were obtained, and $51.4 \%$ of them were annotated in eight public databases. The majority of the 44 candidate genes in the flavonoid biosynthesis pathway were downregulated as leaves aged. Metabolome profiling revealed a set of 427 metabolites in leaves. Consistent with the transcriptome results, 15 of the 19 metabolites in the flavonoid pathway decreased during the development of leaves. The data indicate that young leaf is the optimal stage for tea harvest. This is the first report of integrated transcriptome and metabolome profiling of $L$. polystachyus. This study demonstrates the correlation of gene expression and metabolites related to flavonoid biosynthesis and reveals the key genes responsible for flavonoid accumulation in young leaf. The information can be applied to future studies performed to elucidate and manipulate flavonoid biosynthesis in L. polystachyus.
\end{abstract}

Lithocarpus polystachyus, also known as the sweet tea, is an evergreen tree that can reach a height of $715 \mathrm{~m}$. It belongs to the family Fagaceae and is mainly distributed in the mountain area of southern China, India, and Thailand (Li et al., 2019). Its tender leaves are often collected by local people to make tea, which has anti-diabetic, anti-hypertensive, anti-obesity, and anti-hypoglycemic biological activities (Hou et al., 2011; Zhou et al., 2013).

Plant secondary metabolites are primarily produced in leaves, among which flavonoids constitute a class of important

Received for publication 16 Nov. 2020. Accepted for publication 14 Jan. 2021. Published online 2 March 2021.

K.-X.L., K.L., and Y.C. contributed equally to this work.

We thank the Forestry Public Welfare projects of China (no. 201204612), Guangxi scientific research and technological development projects (no. GKG1598006-5-6), and Guangxi natural science fund projects (no. 2015GXNSFAA139099) for funding in this work.

Y.S. and H.L. are the corresponding authors. E-mail: ybshen@bjfu.edu.cn or hliang@clemson.edu.

This is an open access article distributed under the CC BY-NC-ND license (https://creativecommons.org/licenses/by-nc-nd/4.0/). compounds in herbal medicine (Guo et al., 2020). Flavonoids are the major active secondary metabolites in the leaves of $L$. polystachyus (Li et al., 2008). The many flavonoids that have been identified include phloretin/phlorizin, trilobatin, and quercetin (Wang et al., 2016b). The biological activities of flavonoids are diverse. Numerous studies have demonstrated that the flavonoid fraction extracted from L. polystachyus leaves has an important role in the treatment of diabetes, hypertension, and chronic illness (Hou et al., 2011; Hou et al., 2012). Flavonoids, including phloridzin and trilobatin, have the ability to sustain normoglycemia in type II diabetes mellitus (Dong et al., 2012; Gaucher et al., 2013). Leaf extracts from L. polystachyus promote glycogen synthesis and have enhanced anti-diabetic effects when compared with trilobatin and phloridzin alone ( $\mathrm{Li}$ et al., 2019). Trilobatin from L. polystachyus has strong inhibitory activity against $\alpha$-glucosidase and mild inhibitory activity against $\alpha$-amylase, rendering it a moderate antioxidant activity (Dong et al., 2012). The flavonoid fraction of L. polystachyus has been found to significantly reduce the blood pressure of spontaneously hypertensive rats; therefore, it could potentially 
be used as a natural drug or functional food for preventing hypertension (Hou et al., 2012). Recent studies have shown that flavonoids extracted from L. polystachyus, such as trialosides, have an important role in hindering neuronal death, neuroinflammation, and oxidative stress. This reveals the potential of flavonoids for treating Alzheimer's disease and protecting neuronal cells against neurotoxicity (Chen et al., 2020; Gao et al., 2020; Shen et al., 2020). Therefore, there is substantial interest in understanding the flavonoid metabolism.

Flavonoids are synthesized through a complex pathway that has largely been found in Arabidopsis thaliana and tea plant (Camellia sinensis). In A. thaliana, key enzyme genes involved in flavonoid biosynthesis, including chalcone synthase (CHS), chalcone flavanone isomerase $(\mathrm{CHI})$, flavanone 3-hydroxylase $(F 3 H)$, and flavonol synthase $(F L S)$, are regulated by the AtMYB 12 transcription factor gene, leading to the increased accumulation of flavonoids (Wang et al., 2016a). In tea plants, the major genes in flavonoid biosynthesis, including $F 3 H$, flavone synthase gene (FNS), anthocyanidin 3-O-glucosyltransferase gene (UFGT), and transcription factors genes bHLH35 and $b H L H 36$, are upregulated in nitrogen-deficient conditions (Huang et al., 2018). To date, little information is available regarding flavonoid biosynthesis in L. polystachyus. Zhang et al. (2019) reported that the accumulation of flavonoids in the species was affected by the light quality, light intensity, and photoperiod. In particular, phlorizin accumulation was positively related to the transcription level of several genes encoding phenylalanine ammonia lyase (PAL), 4-coumarate:CoA ligase (4CL), and 4-coumarate-CoA ligase-like (4CLL), suggesting the importance of these genes in phlorizin biosynthesis (Zhang et al., 2019). Harvesting time, location, and leaf age were also found to influence the metabolites and, thus, qualities of sweet tea (Zhou et al., 2013). Yang et al. (2018) reported that the flavonoid content was highest in young leaves and decreased at the mature stage.

RNA sequencing is a powerful tool for unraveling novel genes and identifying genes expressed under different conditions. Metabolomics is an important part of systematic biology that focuses on the quantitative analysis of all metabolites in organisms. Analyses of transcriptomics and metabolomics have contributed to the identification of functional genes and the elucidation of pathways involved in plant metabolism processes (Gao et al., 2019; Guo et al., 2020; Gutierrez et al., 2017; Zhang et al., 2019). In this study, we used this integrated approach to study metabolite pathways associated with flavonoid biosynthesis in $L$. polystachyus. We investigated the gene expression and metabolic differences among young, mature, and old leaves. The data presented here will help form the basis of future studies of the molecular mechanism underlying L. polystachyus flavonoid metabolism. Our study provides useful information for the application of L. polystachyus young leaf in the pharmaceutical industry.

\section{Materials and Methods}

Plant materials. Six-year-old L. polystachyus rooted cuttings grown in the Economic Forest Garden of Guangxi Forestry Research Institute, Guangxi, China (lat. $22^{\circ} 92^{\prime} \mathrm{N}$, long. $108^{\circ} 35^{\prime} \mathrm{E}$ ) were used for this study. The plants belonged to the same cultivar Guiduo1 and were free of pests and diseases. Leaves of different developmental stages were collected separately from each tree on 15 June 2018. Young leaves were 3-dold healthy leaves from the top of 1-year-old branches (LP-1). Mature leaves were 15-d-old healthy leaves collected in the middle of 1-year-old branches (LP-2). Old leaves were dark green, leathery leaves from the base of 1-year-old branches (LP-3). More than three leaves were collected and combined to form one replicate, with a total of three replicates. These materials were immediately frozen in liquid nitrogen after harvest and stored at $-80^{\circ} \mathrm{C}$ for RNA sequencing and metabolic profiling analyses.

TransCRIPTOME SEQUenCing AND ANALYSIS. For each developmental stage, three independent biological replicates were used to extract total RNA with an RNAprep Pure Plant kit (Tiangen, Beijing, China). The RNA purity was checked using a spectrophotometer (NanoPhotometer; Implen, Westlake Village, CA). The RNA concentration was measured with an RNA Assay Kit in a flurometer (Qubit 2.0; Life Technologies, Carlsbad, CA). The RNA integrity was assessed using an RNA Nano 6000 Assay Kit in a bioanalyzer (2100 Bioanalyzer System; Agilent Technologies, Santa Clara, CA). Sequencing libraries were generated with RNA library prep kits (NEBNext Ultra II; New England Biolabs, Ipswich, MA) following the manufacturer's recommendations. Then, the libraries were sequenced on a high-throughput sequencer (HiSEq 2000; Illumina, San Diego, CA) for paired-end reads.

Before assembly, the raw data were filtered by trimming adaptor sequences and removing low-quality sequences $(\mathrm{Q}<$ 20 ) with more than $10 \%$ uncertain $(\mathrm{N})$ bases. The clean reads were assembled de novo using Trinity software (Grabherr et al., 2011). The gene function was annotated based on the following databases: National Center for Biotechnology Information (NCBI, Bethesda, MD) nonredundant protein sequences (NR) (Benson et al., 2010); Protein family (Pfam) (Finn et al., 2014); euKaryotic Orthologous Groups (KOG) (Koonin et al., 2004);

Table 1. Summary of sequencing reads after filtering. For each leaf developmental stage (young leaf: LP-1-1, LP-1-2, LP-1-3; mature leaf: LP-2-1, LP-2-2, LP-2-3; old leaf: LP-3-1, LP-3-2, LP-3-3), three independent biological replicates were collected from Lithocarpus polystachyus and sequenced. The total clean reads were obtained after removing the reads with a linker or lowquality reads. The guanine-cytosine (GC) content indicates the percentage of nitrogenous bases in the sequences that are either guanine $(\mathrm{G})$ or cytosine $(\mathrm{C})$. Q30 represents the percentage of bases with a Phred quality score more than 30 .

\begin{tabular}{lccccc}
\hline Sample & Code & $\begin{array}{c}\text { Total clean } \\
\text { reads (no.) }\end{array}$ & $\begin{array}{c}\text { Total clean } \\
\text { bases (bp) }\end{array}$ & $\begin{array}{c}\text { GC content } \\
(\%)\end{array}$ & Q30 (\%) \\
\hline Young leaves & LP-1-1 & $20,317,668$ & $6,082,195,690$ & 44.37 & 89.60 \\
& LP-1-2 & $23,905,271$ & $7,157,486,058$ & 44.42 & 89.34 \\
Mature leaves & LP-1-3 & $22,627,650$ & $6,764,160,666$ & 44.11 & 89.40 \\
& LP-2-1 & $23,278,028$ & $6,971,077,864$ & 44.16 & 88.76 \\
& LP-2-2 & $21,394,671$ & $6,402,081,748$ & 44.05 & 89.32 \\
Old leaves & LP-2-3 & $21,506,781$ & $6,435,475,216$ & 44.16 & 88.83 \\
& LP-3-1 & $22,318,047$ & $6,686,917,630$ & 44.16 & 89.68 \\
& LP-3-2 & $21,480,578$ & $6,433,949,202$ & 44.45 & 89.77 \\
& LP-3-3 & $22,755,379$ & $6,815,664,498$ & 44.68 & 89.09 \\
\hline
\end{tabular}



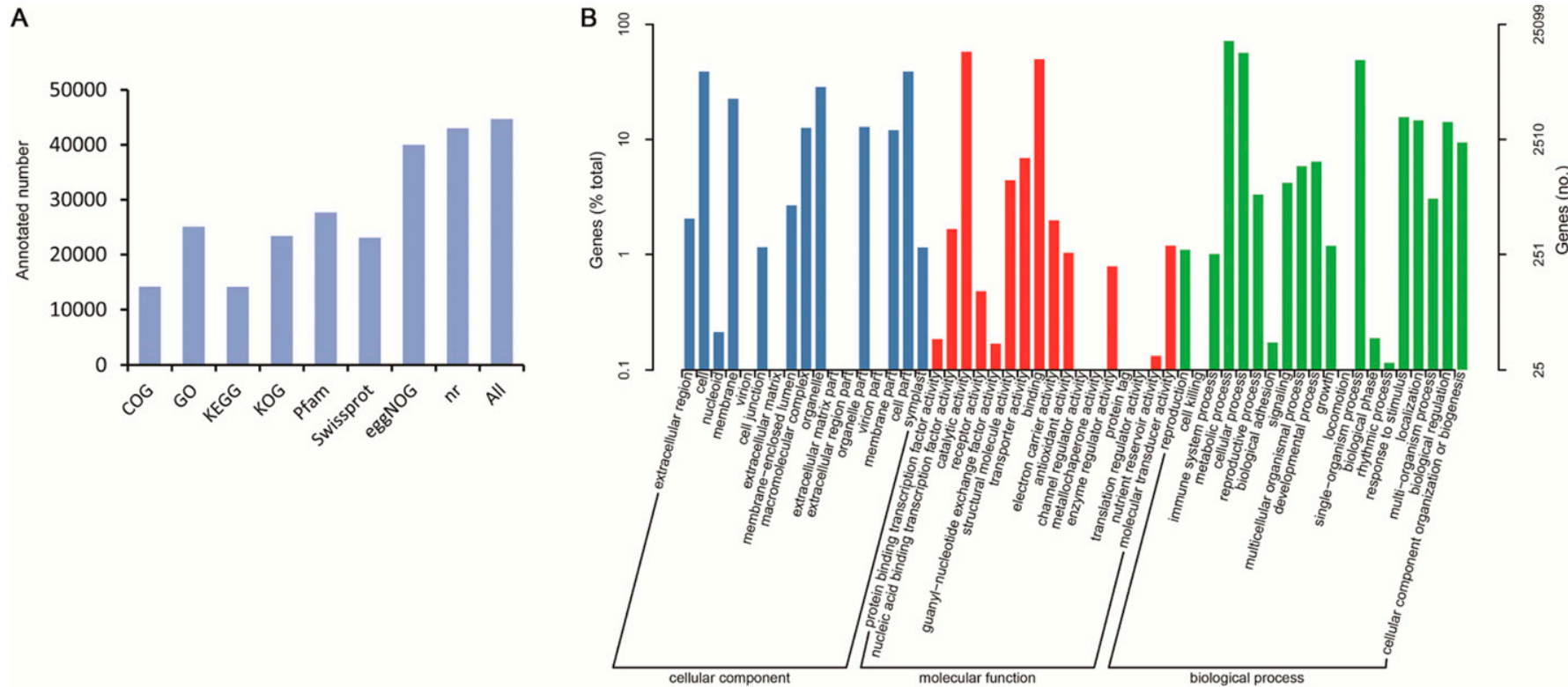

Fig. 1. Unigene annotation results of Lithocarpus polystachyus leaf transcriptome. (A) Annotation information obtained from eight different databases: Clusters of Orthologous Groups (COG); Gene Ontology (GO); Kyoto Encyclopedia of Genes and Genomes (KEGG); euKaryotic Orthologous Groups (KOG); Protein family (Pfam); a manually annotated and reviewed protein sequence database (Swiss-Prot); evolutionary genealogy of genes: nonsupervised Orthologous Groups (eggNOG); and nonredundant protein sequences (NR). (B) Annotation information based on the GO database.

Table 2. Differentially expressed genes (DEGs) in pairwise comparisons of young, mature, and old leaves of Lithocarpus polystachyus. Upregulated and downregulated genes are listed separately for each comparison.

\begin{tabular}{lccc}
\hline Comparison & $\begin{array}{c}\text { All DEGs } \\
\text { (no.) }\end{array}$ & $\begin{array}{c}\text { Upregulated } \\
\text { DEGs (no.) }\end{array}$ & $\begin{array}{c}\text { Downregulated } \\
\text { DEGs (no.) }\end{array}$ \\
\hline Young vs. mature & 3,187 & 1,457 & 1,730 \\
Young vs. old & 5,886 & 2,521 & 3,365 \\
Mature vs. old & 3,794 & 1,213 & 2,581 \\
\hline
\end{tabular}

with three biological replicates and relative expression levels were calculated based on the $2^{-\Delta \Delta \mathrm{Ct}}$ method (Livak and Schmittgen, 2001).

METABOLOMIC PROFILING ANALYSIS. The freeze-dried leaves were crushed and the fine powder $(100 \mathrm{mg})$ was weighted and extracted overnight at $4{ }^{\circ} \mathrm{C}$ with $0.6 \mathrm{~mL}$ $70 \%$ aqueous methanol. Following

Clusters of Orthologous Groups (COG) (Tatusov et al., 2001); Evolutionary genealogy of genes: nonsupervised Orthologous Groups (eggnog) (Huerta-Cepas et al., 2017); a manually annotated and reviewed protein sequence database (Swiss-Prot) (Boeckmann et al., 2003); Kyoto Encyclopedia of Genes and Genomes (KEGG) (Kanehisa et al., 2004); and Gene Ontology (GO) (Ashburner et al., 2000). A differential expression analysis of different developmental stages was performed using the DESeq R package (1.10.1) (Anders and Huber, 2010). The resulting probability values were adjusted using the approach of Benjamini and Hochberg (1995) for controlling the false discovery rate. Differentially expressed genes (DEGs) were defined with a threshold of $\mid \log _{2}$ (fold-change) $\mid \geq 1$ and false discovery rate $($ FDR $) \leq 0.01$. Sequence data generated for this study were deposited in the NCBI Sequence Read Archive (SRA) (Leinonen et al., 2011) database (BioProject ID PRJNA670041).

QUANTITATIVE REVERSE-TRANSCRIPTION POLYMERASE CHAIN REACTION. The expression of selected candidate genes was determined by quantitative reverse-transcription polymerase chain reaction (qRT-PCR). Gene-specific primers were designed for 10 randomly selected candidate genes based on the obtained sequences. The actin gene (c95882.graph_c0) was used as a reference. Primers used for qRT-PCR are listed in Supplemental Table 1. The qRT-PCR assay was performed centrifugation at $10,000 g_{\mathrm{n}}$ for $10 \mathrm{~min}$, the extracts were absorbed and filtrated before ultra-fast liquid chromatography combined with electrospray ionization tandem mass spectrometry (UFLC-ESI-MS/MS). The sample extracts were analyzed using an UFLC-ESI-MS/MS system (UFLC: Shim-pack UFLC CBM30A system, Shimadzu, Kyoto, Japan; MS: 4500 Q TRAP system, Applied Biosystems, Foster, CA). To obtain data that could be evaluated for repeatability, the quality controls (QCs) were injected in every five samples to identify potential sample carryover. Linear ion trap (LIT) and triple quadrupole (QQQ) scans were performed using a triple quadrupole-linear ion trap mass spectrometer (Q TRAP; API 4500 Q TRAP UFLC/MS/ MS System) equipped with an electrospray ionization Turbo Ion-Spray interface and operated using the positive and negative ion modes. Analyst 1.6.3 (AB Sciex, Concord, ON, Canada) software was used.

An unsupervised principal component analysis (PCA) was performed using statistics function prcomp in R (R Core Team, 2014). The hierarchical cluster analysis (HCA) results were presented as heatmaps with dendrograms, and Pearson correlation coefficients (PCCs) were calculated by the cor function in $\mathrm{R}$ and presented as heatmaps. The detection of differential metabolites was determined by variable influence on projection (VIP) $\geq 1$ and $\mid \log _{2}$ (fold-change) $\mid \geq 1$. The VIP values were extracted from the orthogonal projection based on the latent 
structure-discriminant analysis (OPLS-DA) results, which also included score plots and permutation plots. The OPLS-DA combines orthogonal signal correction (OSC) and projection based on the latent structure-discriminant analysis, which can decompose the X-matrix information to Y-relevant and $\mathrm{Y}$ irrelevant and select the different variables by removing the irrelevant differences. The identified metabolites were annotated using the KEGG Compound database (Hattori et al., 2010).

INTEGRATED TRANSCRIPTOME AND METABOLOME ANALYSIS. TO examine the associations between gene expression and metabolite content, we conducted Pearson correlation tests of the discriminant gene expression and metabolite content. Associations between the Pearson correlation coefficient $(\mathrm{PCC})>0.8$ and $P<0.05$ were selected and visualized using Cytoscape software version 3.7.2 (Cytoscape Consortium, San Diego, CA). In addition, DEGs and differentially accumulated metabolites in the flavonoid biosynthesis pathway (ko00941) were mapped to the KEGG pathway database.

\section{Results}

Transcriptome anAlysis and differently EXPRESSED GENES. A total of nine RNA libraries for three different developmental stages of L. polystachyus leaves were analyzed. LP-11, LP-1-2, and LP-1-3 were from young leaves, LP-2-1, LP-2-2, and LP-2-3 were from mature leaves, and LP-3-1, LP-3-2, and LP-3-3 were from old leaves. When the correlation value was calculated using the quantitative results of FPKM among samples, it was found that samples from the same time point had a correlation value of at least 0.9 , indicating high consistency between the biological replicates (Supplemental Fig. 1). After removing the reads with a linker or low-quality reads, a range of 20.32 to 23.91 million clean reads comprising 6.08 to $7.16 \mathrm{~Gb}$ were obtained for each library. The guanine-cytosine (GC) content of the sequences for the samples was $44.3 \%$, and more than $88 \%$ of the bases had a Phred quality score more than 30 , indicating high quality and accuracy of the high-throughput sequencing (Table 1). Assembly of the clean reads resulted in a total of 86,927 unigenes (73.5 Mb of sequences), which had a mean length of $846 \mathrm{bp}$ and an N50 length of $1616 \mathrm{bp}$.

The BLAST alignment with eight public databases (NR, Swiss-Prot, GO, COG, KOG, eggNOG, Pfam, and KEGG) identified 44,696 unigenes with annotation in at least one of the databases, accounting for $51.4 \%$ of all unigenes. Among them, 43,035 unigenes (49.5\% of all unigenes) had significant matches to the NR database, which was the most matched database. Regarding the other databases, there were 40,005 (46.0\%), 27,714 (31.9\%), 25,099 (28.9\%), 23,391 (26.9\%), 23,101 (26.6\%), 14,179 (16.3\%), and 14,146 (16.3\%) significant hits in eggNOG, Pfam, GO, KOG, Swiss-Prot, KEGG, and COG, respectively (Fig. 1A). Figure 1B and Supplemental Table 2 show the GO annotations in terms of the cellular component (CC), biological process (BP), and molecular function $(\mathrm{MF})$. Within the $\mathrm{CC}$ category, the unigenes matched 17 GO terms, with "cell" (9768), "cell part" (9767), and "organelle" (7180) representing the predominant terms. Regarding the BP category, the unigenes matched $20 \mathrm{GO}$ terms, with "metabolic process" $(18,052)$, "cellular process" $(14,205)$, and "single-organism process" (12,313) representing the main terms. In the MF category, the unigenes matched 17 GO terms, with "catalytic activity" (14,509), "binding"
$(12,497)$, and "transporter activity" (1731) as the top three terms. In the KEGG annotation, the unigenes were assigned to 129 KEGG pathways. Among them, "ribosome" (1081), "carbon metabolism" (816), and "biosynthesis of amino acids" (609) pathways contained the majority of the unigenes. A set of 44 unigenes were annotated in the "flavonoid biosynthesis" pathway. Transcription dynamics among the three different developmental stages of leaves were examined according to the fragments per kilobase of transcript per million (FPKM) mapped reads. A total of 3187, 5886, and 3794 differently expressed genes $\left[F D R<0.01\right.$ and $\mid \log _{2}$ (foldchange) $\mid \geq 1$ ] were found during the comparisons of young and mature leaves, young and old leaves, and mature and old leaves, respectively (Table 2). Among these DEGs, 681 genes were differently regulated during all three comparisons. Totals of 511,1658 , and 639 genes were uniquely found during the comparisons of young and mature leaves, young and old leaves, and mature and old leaves, respectively (Fig. 2).

IDENTIFICATION OF UNIGENES RELATED TO FLAVONOID BIOSYNTHESIS. The L. polystachyus leaf transcriptome obtained 44 candidate genes in the flavonoid biosynthesis pathway (ko00941). Among these genes, 10 were significantly downregulated in mature leaves compared to young leaves and three were significantly upregulated. Compared with mature leaves, all 14 DEGs in old leaves were downregulated. Compared with young leaves, old leaves had 17 downregulated genes and one upregulated gene. These data indicated that the majority of the DEGs in the flavonoid biosynthesis pathway were downregulated as leaves aged (Fig. 3, Supplemental Table 3). The enzymes encoded by these downregulated unigenes included CHI (c103291.graph_c0), transcinnamate 4-monooxygenase

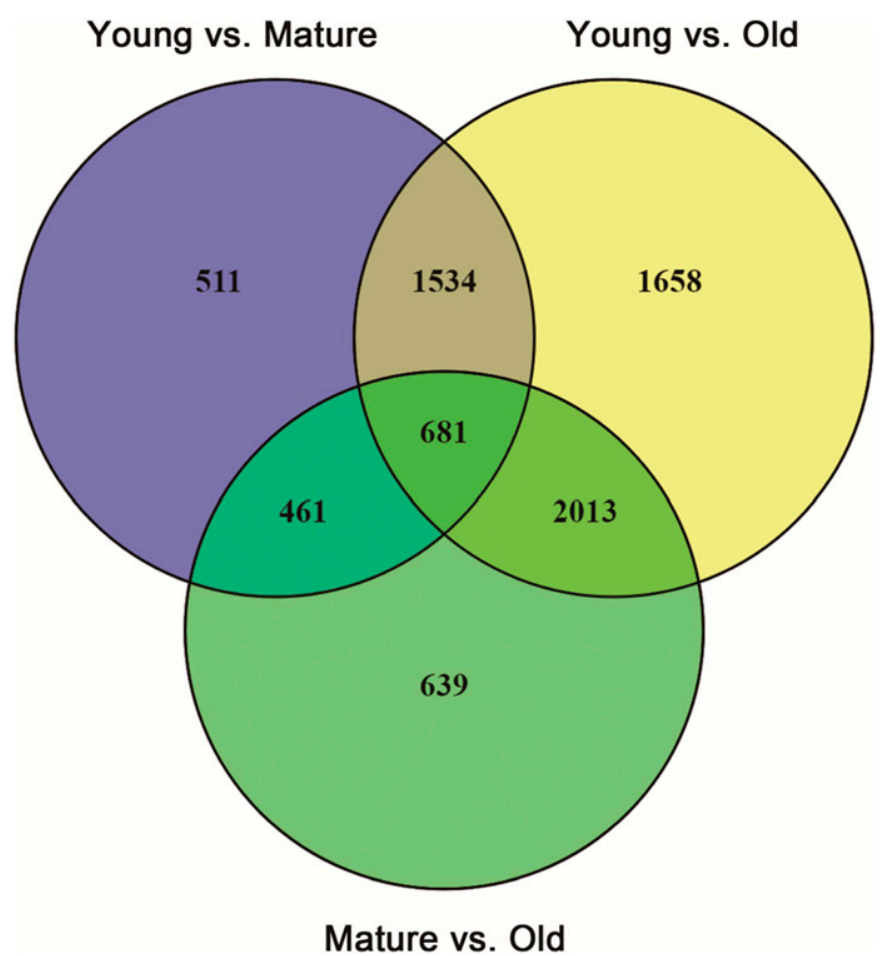

Fig. 2. Venn diagram of differentially expressed genes (DEGs) during pairwise comparisons of young, mature, and old leaves of Lithocarpus polystachyus. 


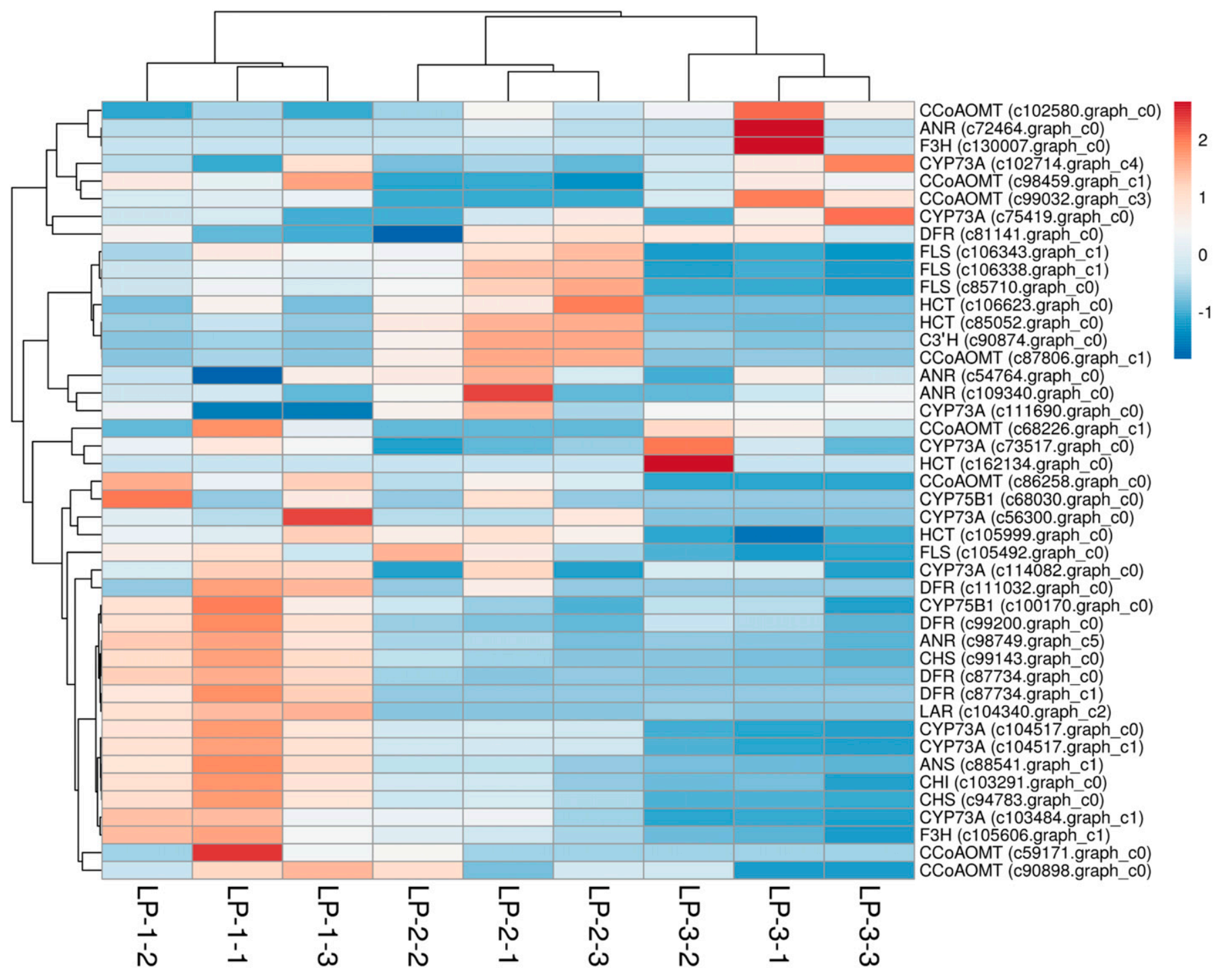

Fig. 3. Expression heatmap of 44 unigenes in the flavonoid biosynthesis pathway based on a hierarchical clustering analysis of young (LP-1), mature (LP-2), and old (LP-3) leaves of Lithocarpus polystachyus. Red and blue, respectively, indicate high and low expression according to the color bar using the unit variance scaling method.

(CYP73A; c104517.graph_c1, c103484.graph_c1, c104517. graph_c0), leucoanthocyanidin dioxygenase (ANS; c88541. graph_c1), F3H (c105606.graph_c1), FLS (c106338.graph_c1, c106343.graph_c1, c85710.graph_c0), caffeoyl-CoA O-methyltransferase (CCoAOMT; c86258.graph_c0), flavonoid 3'-monooxygenase (CYP75B1; c100170.graph_c0), leucoanthocyanidin reductase (LAR; c104340.graph_c2), anthocyanidin reductase (ANR; c98749.graph_c5), CHS (c99143.graph_c0, c94783.graph_c0), and flavanone 4-reductase (DFR; c99200. graph_c0, c87734.graph_c0). Three unigenes were upregulated in the mature leaves and downregulated in the old leaves. These unigenes putatively encode shikimate O-hydroxycinnamoyltransferase (HCT; c85052.graph_c0), CCoAOMT (c87806.graph_c1), and coumaroylquinate $3^{\prime}$-monooxygenase $\left(\mathrm{C} 3^{\prime} \mathrm{H}\right.$; c90874.graph_c0) enzymes in the lignin biosynthesis pathway.

QRT-PCR VALIDATION OF DIFFERENTLY EXPRESSED GENES. TO verify the reliability of the transcriptome data, the transcript level of 10 unigenes was evaluated by qRT-PCR with three biological replicates. Nine of these unigenes displayed expression trends that were similar to those obtained during the RNASeq analysis, corroborating the RNA sequencing data. These include genes encoding CCoAOMT (c86258.graph_c0), HCT (c85052.graph_c0), C3' H (c90874.graph_c0), phosphoglycerate dehydrogenase (c98353.graph_c0), monooxygenase (c89114.graph_c0), and galactose oxidase (c93933.graph_c0). The high consistency of RNA-Seq and qRT-PCR results suggested that the RNA-seq data were reliable for evaluating the regulation of gene expression during different developmental stages of L. polystachyus leaves (Fig. 4).

Metabolomic PROFILING ANALYSIS AND DIFFERENTLY aCCUMULATED METABolites. The compositions of essential metabolites in leaves in different developmental stages were determined by UFLC-MS/MS. A total of 427 metabolites were detected and grouped into 12 categories (Fig. 5A, Supplemental Table 4). The largest category (flavonoids) consisted of 115 metabolites, followed by amino acids and derivatives $(n=63)$, lipids $(n=58)$, and phenolic acids $(n=45)$. The principal 
A

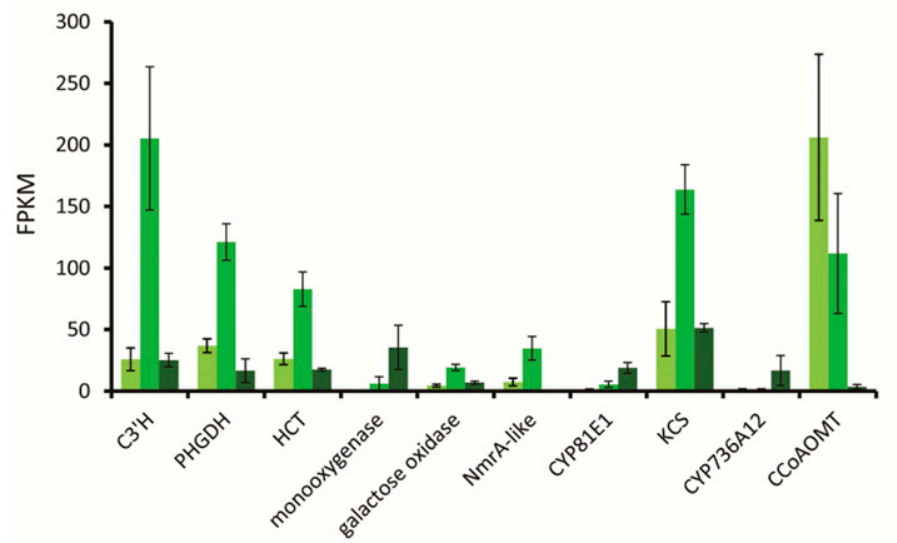

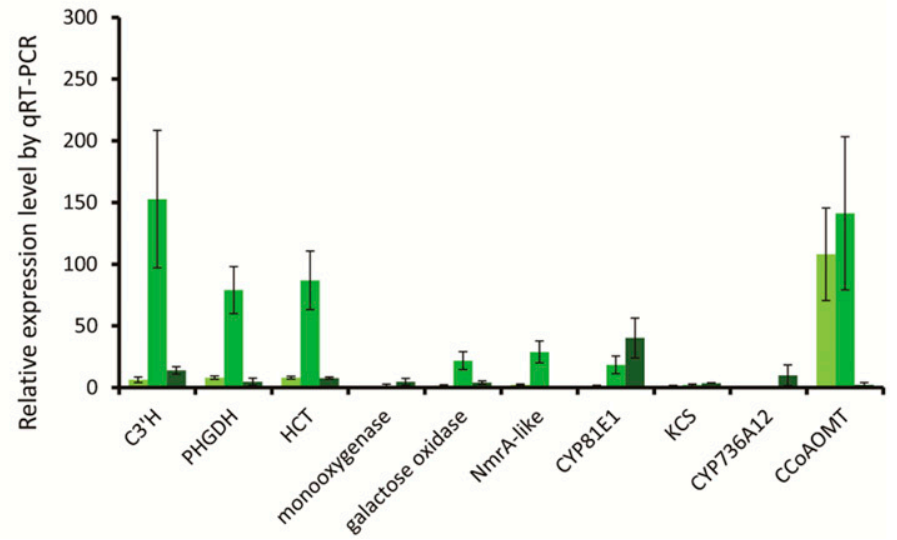

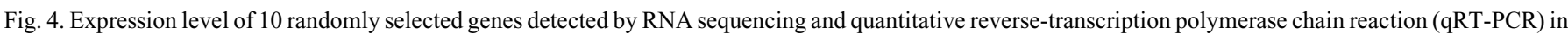
young (light green), mature (green), and old (dark green) leaves of Lithocarpus polystachyus. (A) Expression patterns were based on the fragments per kilobase of transcript per million (FPKM) mapped reads. (B) The qRT-PCR expression levels were normalized with actin. The error bars represent the sD of three replicates.

component analysis (PCA) of metabolites grouped nine samples into three distinct clusters, thereby accurately reflecting the three developmental stages of leaves (Fig. 5B). In the model, $43.4 \%$ and $18.19 \%$ of the total variance could be explained by PC1 and PC2, respectively. Consistently, the hierarchical clustering and correlation analysis showed strong dependence of leaf metabolites on the developmental stages of leaves (Fig. $5 \mathrm{C})$. The overall accumulation of flavonoids was gradually decreased during the development of leaves.

An OPLS-DA model was generated to identify the metabolites responsible for metabolic differentiations among developmental stages of leaves. Totals of 109, 214, and 188 differently accumulated metabolites (VIP $\geq 1$ and $\mid \log _{2}$ (foldchange) $\mid \geq 1$ ) were found during the comparisons of young and mature leaves, young and old leaves, and mature and old leaves, respectively. The number of upaccumulated metabolites and downaccumulated metabolites of each comparison are listed in Table 3. For example, cyanidin 3-O-glucoside (Kuromanin) was abundant in young leaves and downregulated in mature and old leaves. On the contrary, cynaroside was significantly upregulated in old leaves (Supplemental Table 4).

IDENTIFICATION OF METABOLITES RELATED TO FLAVONOID BIOSYNTHESIS. Based on the KEGG database, a total of 19 metabolites were found in the flavonoid biosynthesis pathway (Supplemental Table 4). Among them, three chalcones (naringenin chalcone, 4,2' $4^{\prime}, 6^{\prime}$-tetrahydroxychalcone, phloretin), four dihydroflavones (butin, hesperetin 7-o-neohesperidoside, naringenin, naringin), four flavanols [(-)-epiafzelechin, (-)-epigallocatechin, (+)-gallocatechin, l-epicatechin], two flavonoids (luteolin, tricetin), two flavonoid carbonosides (apigenin-8-c-glucoside, vitexin), two flavonols (kaempferol, quercetin), and two phenolic acids (3-o-p-coumaroyl quinic acid, chlorogenic acid) were found. Interestingly, the contents of 15 of the 19 metabolites decreased during the development of leaves (Fig. 6), suggesting these metabolites were accumulated in the young leaves. Only quercetin and chlorogenic acid were accumulated in the mature leaves. The reduction of the metabolites in the flavonoid biosynthesis pathway is consistent with the downregulation of genes in the flavonoid biosynthesis pathway during the development of leaves.
INTEGRATED TRANSCRIPTOME AND METABOLOME ANALYSIS. The network analyses of DEGs and differently accumulated metabolites were performed in the flavonoid biosynthesis pathway. Positive correlations between the gene expression level and total flavonoid content were found. In the networks, genes and metabolites were highly connected (Fig. 7). When comparing the young leaves with mature leaves, a total of 99 significant correlations were found, including 67 positive correlations and 32 negative correlations. For example, a $\mathrm{CHI}$ unigene, c103291.graph_c0, had significant correlations with 10 metabolites in the flavonoid pathway, and seven of the correlations were positive. A CHS unigene (c99143.graph_c0) had significant correlations with seven metabolites in the flavonoid pathway, and six of them were positive. When comparing the young leaves with old leaves, a total of 237 significant correlations were found, among which 228 were positive. When comparing the mature leaves with old leaves, a total of 143 significant correlations were found and all of them were positive. The results showed that positive correlations existed among most of the genes and metabolites in the flavonoid biosynthesis pathway.

\section{Discussion}

Lithocarpus polystachyus is a traditional medicinal plant that is known to produce sweet tea. The health benefits of L. polystachyus include anti-diabetic, anti-hypertensive, antiobesity, and anti-hypoglycemic biological activities (Hou et al., 2011; Zhou et al., 2013). Flavonoids are secondary metabolites that have a relevant role in flavor and health benefits. The biosynthesis of flavonoids is affected by the age of the leaves (Guo et al., 2020). The quality of sweet tea is greatly influenced by the harvesting time and leaf age. However, little is known about the underlying mechanisms of changes in the flavonoid content at different developmental stages of leaves. The first study to explore flavonoid biosynthetic pathway genes in $L$. polystachyus was reported by Song et al. (2017); during that study, 41,043 unigenes were obtained during RAN sequencing of young leaves and 28 flavonoid biosynthesis-associated unigenes were revealed. Huang et al. (2018) examined the effects of light quality, light intensity, and photoperiod on 
A

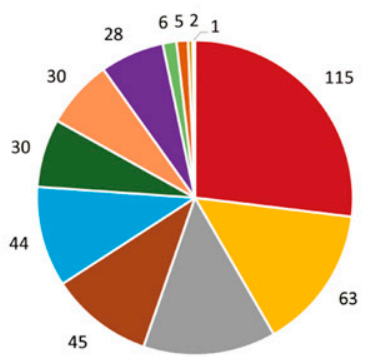

58

\begin{tabular}{|c|c|}
\hline - Flavonoids & $=$ Amino acids and derivatives \\
\hline = Lipids & " Phenolic acids \\
\hline = Others & - Alkaloids \\
\hline = Nucleotides and derivatives & - Organic acids \\
\hline = Tannins & = Lignans and Coumarins \\
\hline = Terpenoids & = Quinones \\
\hline
\end{tabular}

B

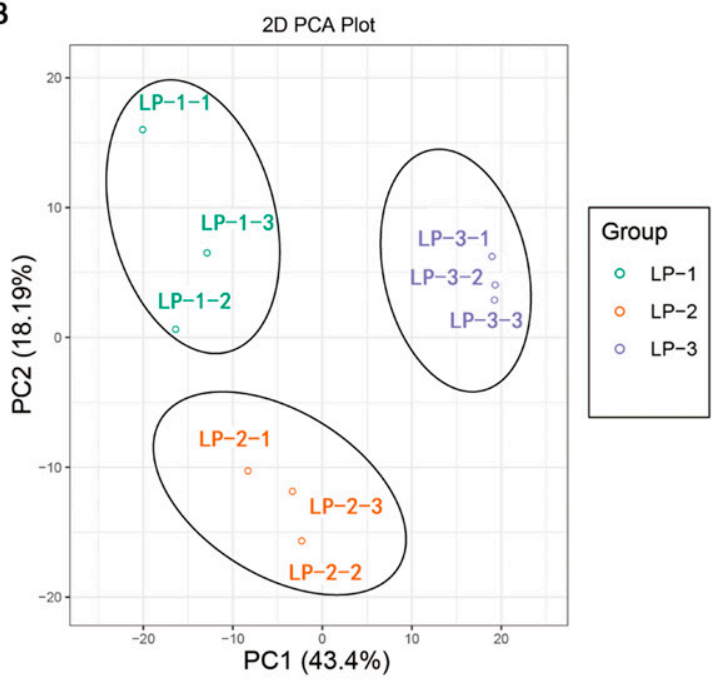

C

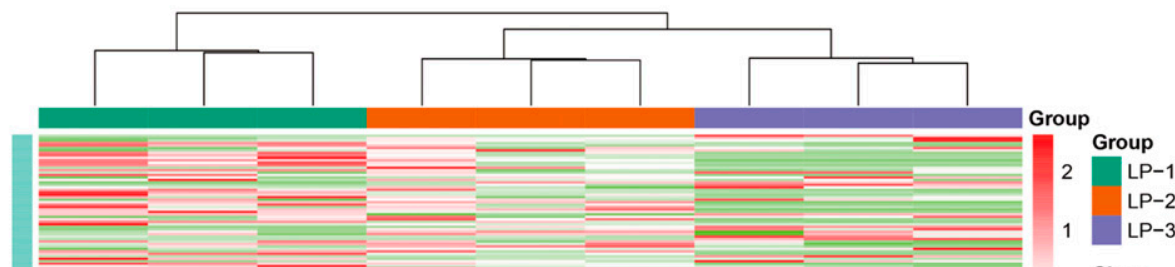

Class

0 Flavonoids

- Amino acids and derivatives

Lipids

Phenolic acids

Others

Alkaloids

Nucleotides and derivatives

Organic acids

Tannins

Lignans and Coumarins

Terpenoids

Quinones

Fig. 5. Metabolomic profiling of young (LP-1), mature (LP-2), and old (LP-3) leaves of Lithocarpus polystachyus. (A) Primary classification of the metabolites identified in the leaves. (B) Principal component analysis (PCA) score plot of young, mature, and old leaves along the first component (PC1) and second component (PC2). (C) Hierarchical clustering and correlation analysis of the metabolites of young, mature, and old leaves.

phlorizin accumulation in the species and focused on lignin biosynthesis genes. Our study was undertaken to augment the knowledge of flavonoid biosynthesis and accumulation at different developmental stages of leaves of L. polystachyus.
The transcriptome analysis of different developmental stages of leaves provided information about the gene expression and regulation related to flavonoid biosynthesis (Li et al., 2012; Liu et al., 2019). Our results showed that the majority of 
Table 3. Number of differentially accumulated metabolites in pairwise comparisons of young, mature, and old leaves of Lithocarpus polystachyus. Upaccumulated and downaccumulated metabolites are listed separately for each comparison.

\begin{tabular}{lccc}
\hline Comparison & $\begin{array}{c}\text { Differently accumulated } \\
\text { metabolites (no.) }\end{array}$ & $\begin{array}{c}\text { Upaccumulated } \\
\text { metabolites (no.) }\end{array}$ & $\begin{array}{c}\text { Downaccumulated } \\
\text { metabolites (no.) }\end{array}$ \\
\hline Young vs. mature & 109 & 66 & 43 \\
Young vs. old & 214 & 157 & 57 \\
Mature vs. old & 188 & 144 & 44 \\
\hline
\end{tabular}

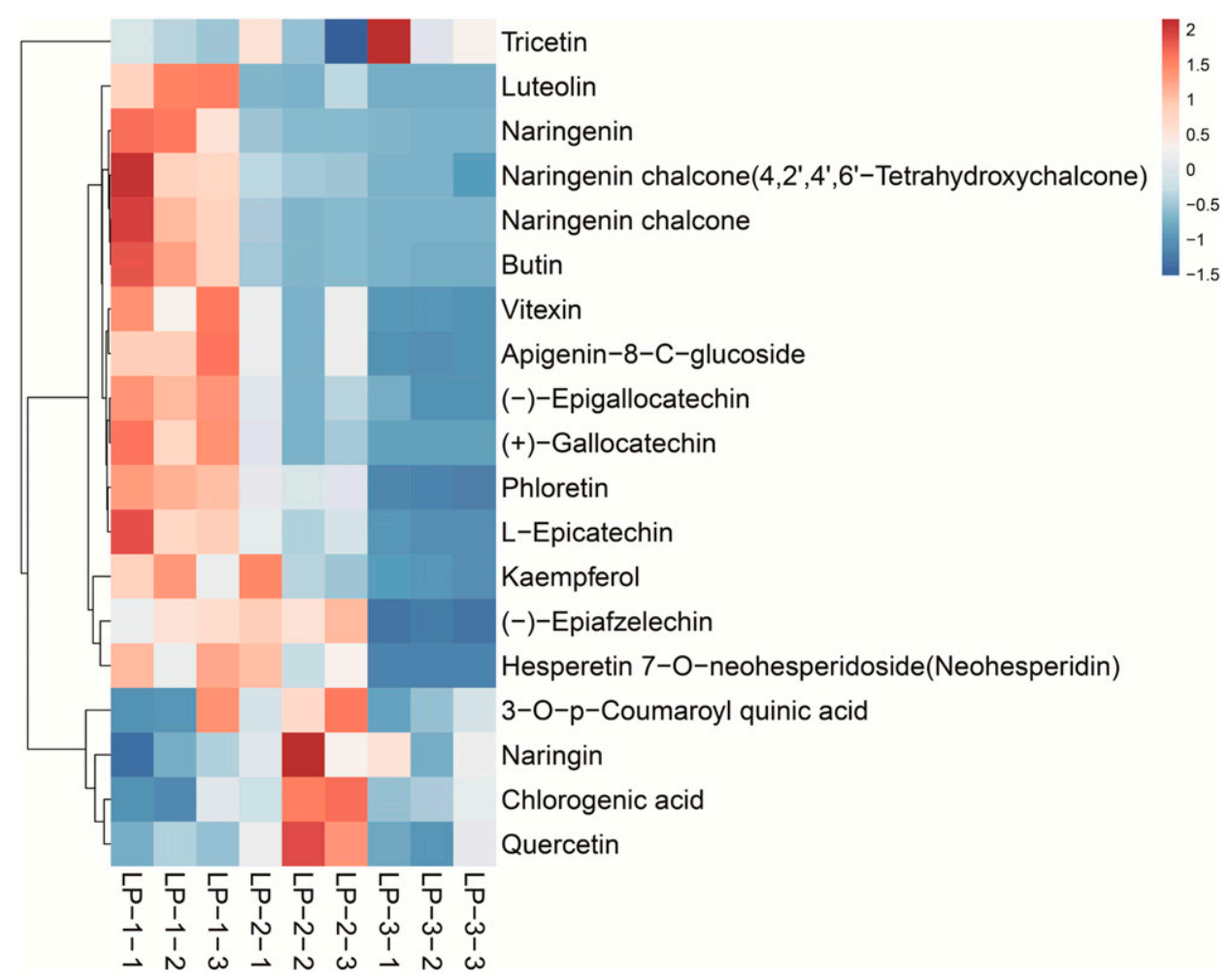

Fig. 6. Hierarchical clustering and correlation analysis of metabolites in the flavonoid biosynthesis pathway of Lithocarpus polystachyus young (LP-1), mature (LP-2), and old leaves (LP-3). et al., 2005). Multiple genes were found encoding the same enzyme in L. polystachyus. For example, three FLS encoding genes were identified. Consistent with the report by Zhang et al. (2019), a set of 4 PAL and 17 4CL encoding genes were identified during the current study (data not shown). Two PAL unigenes (c100908.graph_c0, c100447. graph_c0) were significantly downregulated during the development of leaves, whereas the expression of the other two did not significantly change (c188144.graph_c0, c119242.graph_c0). Three of the $4 C L$ unigenes showed significant changes during the development of leaves (c99603.graph_c0, c102299. graph_c2, and c99089.graph_c1), and they were all downregulated. Because PAL and 4CL enzymes are involved in the upstream steps of the flavonoid biosynthetic pathway, these results suggest that genes involved in the upstream steps of the flavonoid biosynthetic pathway seem to be downregulated in mature and old leaves, consistent with the genes in the flavonoid biosynthetic pathway.

DEGs in the flavonoid biosynthesis pathway were downregulated with the development of the leaf. The precursor of the flavonoid biosynthesis pathway is p-coumaroyl-CoA, which also acts as a bridge connecting primary metabolism and secondary metabolism (Loke et al., 2017). p-coumaroyl-CoA is catalyzed by CHS into chalcone. The gene encoding CHS was highly expressed in the young leaves but downregulated by $\approx 5.3$-times and $\approx 15.3$-times in mature and old leaves, respectively. Consistently, the genes encoding $\mathrm{CHI}$, which are in the second step of the flavonoid biosynthesis pathway, were also significantly downregulated in mature and old leaves. The following steps are catalyzed by F3H and FLS. Interestingly, the expression levels of genes encoding F3H and FLS were comparable in young and mature leaves, but they were significantly downregulated by at least 4.7 -times in old leaves. The findings of the present study suggest that the first few steps of flavonoid biosynthesis are tightly and coordinatively regulated at different developmental stages of leaves. It should be noted that multiple unigenes may be annotated as the same enzyme, mainly because these unigenes belong to different selective splicing transcripts as well as a specific gene family (Yang
Consistent with the transcriptome results, the content of naringenin chalcone, which is the catalytic product of CHS, decreased by 14.6- and 391.8-times in mature and old leaves, respectively. Furthermore, $\mathrm{CHI}$ catalyzes naringenin chalcone into naringenin, which also decreased by 18.1 - and 219.5-times in mature and old leaves, respectively. An overexpression of CHS coupled to CHI in young leaves is consistent with the higher concentration of naringenin chalcone and naringenin, and it supports the active synthesis therein. Naringenin chalcone and naringenin have been found to be useful alternatives for the topical treatment of inflammatory and allergic skin disorders (Escribano-Ferrer et al., 2019). Naringenin chalcone is abundant in the diet, and its anti-cancer effect is also observed in human glioblastoma cells and in the xenograft mice model (Zhang et al., 2016). Beneficial effects of naringenin have been shown for nonalcoholic fatty liver disease (NAFLD) through the regulation of lipid metabolism (Guo et al., 2020; Hernández-Aquino and Muriel, 2018). The most abundant metabolite in young $L$. polystachyus leaves is neohesperidin, which has a potent anti-diabetic effect and is a potential anti-aging flavonoid (Guo et al., 2019). Phloretin is 


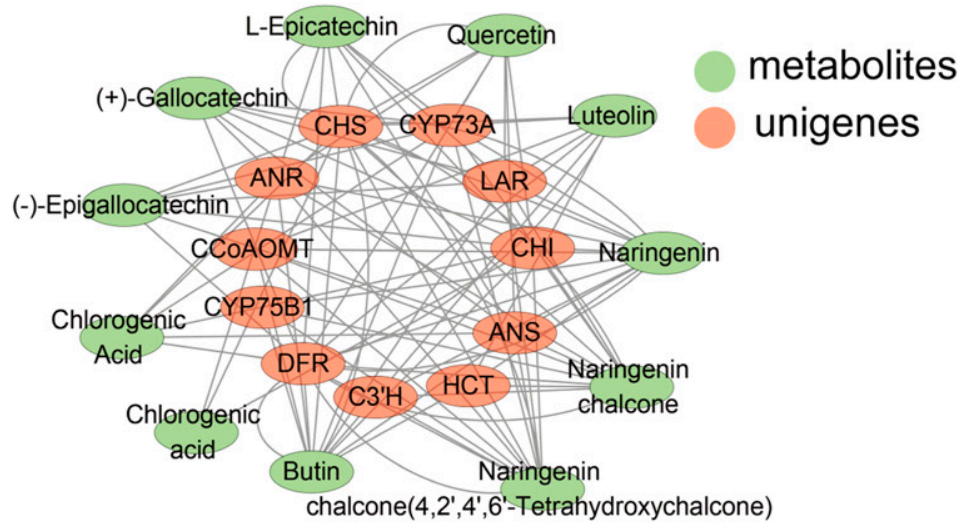

Young vs. Mature

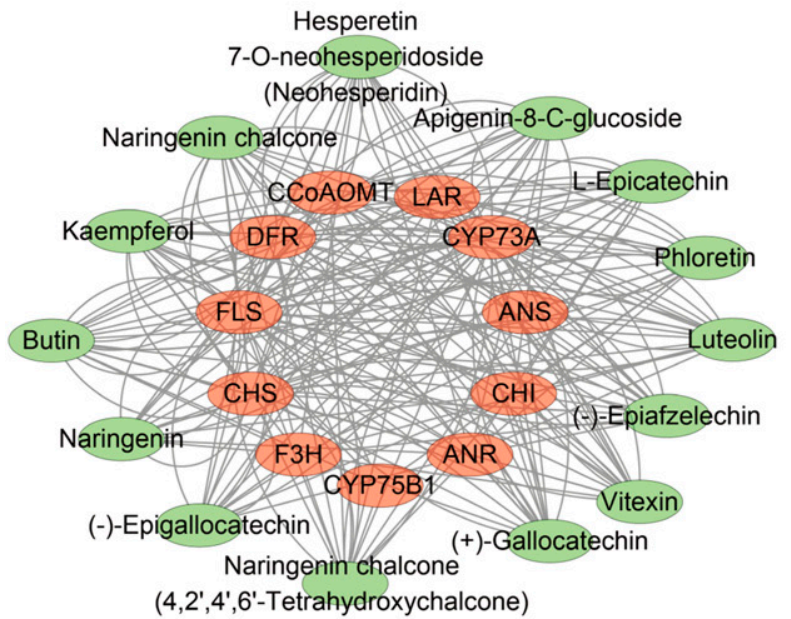

Young vs. Old

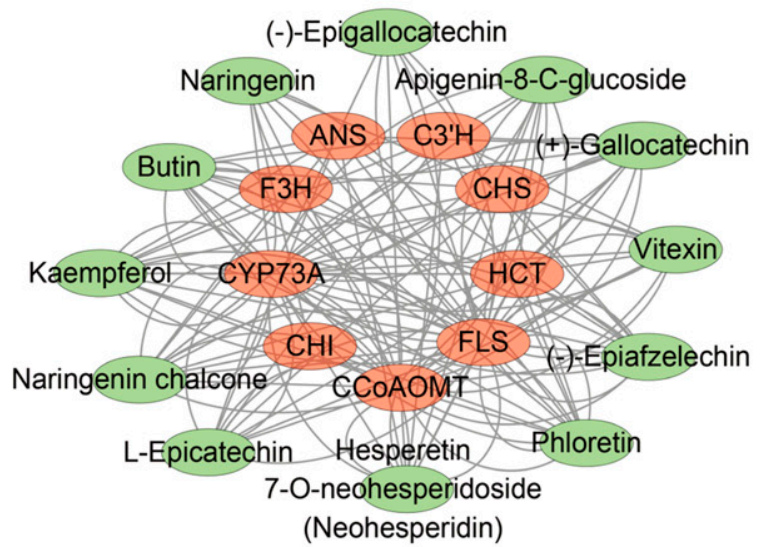

Mature vs. Old

Fig. 7. Results of the correlation analysis of differentially expressed genes (DEGs) and differently accumulated metabolites in the flavonoid biosynthesis pathway based on pairwise comparisons of young, mature, and old leaves of Lithocarpus polystachyus. The correlations between unigenes and metabolites are indicated by a grey line $(\mathrm{CHS}=$ chalcone synthase; $\mathrm{CHI}=$ chalcone flavanone isomerase; $\mathrm{F} 3 \mathrm{H}=$ flavanone 3-hydroxylase; $\mathrm{FLS}=$ flavonol synthase; $\mathrm{ANR}=$ anthocyanidin reductase; ANS $=$ leucoanthocyanidin dioxygenase; $\mathrm{DFR}=$ flavanone 4-reductase; CYP75B1 = flavonoid $3^{\prime}$-monooxygenase; CCoAOMT = caffeoyl-CoA O-methyltransferase; $\mathrm{CYP} 73 \mathrm{~A}=$ transcinnamate 4-monooxygenase; $\mathrm{HCT}=$ shikimate $\mathrm{O}$-hydroxycinnamoyltransferase; $\mathrm{C}^{\prime}{ }^{\prime} \mathrm{H}=$ coumaroylquinate 3 '-monooxygenase; LAR = leucoanthocyanidin reductase). the direct precursor of phlorizin, which is the main sweet ingredient of $L$. polystachyus. Phloretin has various pharmacological effects that can reduce the risk of diabetic complications, lower the blood cholesterol level, and inhibit the development of various cancers (Mariadoss et al., 2019). The content of phloretin was significantly enriched in young leaves. Because of the accumulation of these effective metabolites in young leaves, sweet tea is made from young leaves. Our study corroborates the results of other reports indicating that various metabolites are produced as the intermediate or final products during the growth and development of leaves (Guo et al., 2020; Liu et al., 2019; Rubel Mozumder et al., 2020; Tajidin et al., 2019). In wild soybean (Glycine soja) plants, the nitrogen metabolism pathway was significantly enhanced in old leaves compared with young leaves (Liu et al., 2019). In Andrographis paniculate, a comparison of the leaf stage indicated that young leaves were separated from mature leaves due to the larger quantities of andrographolide (ANDRO), neoandrographolide (NAG), 14-deoxyandrographolide (DAG), glucose, and sucrose (Tajidin et al., 2019). Because of the higher abundance of flavonoids in young $L$. polystachyus leaves than in mature and old leaves, young leaf is the optimal stage for tea harvest.

During this study, we sequenced and analyzed the transcriptome and metabolome at three developmental stages of leaves. Young, mature, and old leaves of L. polystachyus showed dramatic differences in the gene expression levels of the flavonoid biosynthesis pathway. The enzymes catalyzing the first few steps of flavonoid biosynthesis, including $\mathrm{CHS}, \mathrm{CHI}, \mathrm{F} 3 \mathrm{H}$, and FLS, were largely significantly downregulated during the development of leaves. Consistent with the transcriptome results, the content of most metabolites in the flavonoid pathway decreased during the development of leaves. Effective metabolites, including naringenin chalcone, naringenin, neohesperidin, and phloretin, were significantly enriched in young leaves. Therefore, the present study demonstrated how the gene expression and metabolite accumulation changed during the leaf development of $L$. polystachyus. Figure 8 summarize the results obtained from transcriptome and metabolome profiling. This study demonstrated that global transcriptomic programming and metabolomic reprogramming have key roles in the accumulation of flavonoids, thus leading to the sweet tea flavor and potential therapeutic effects. 


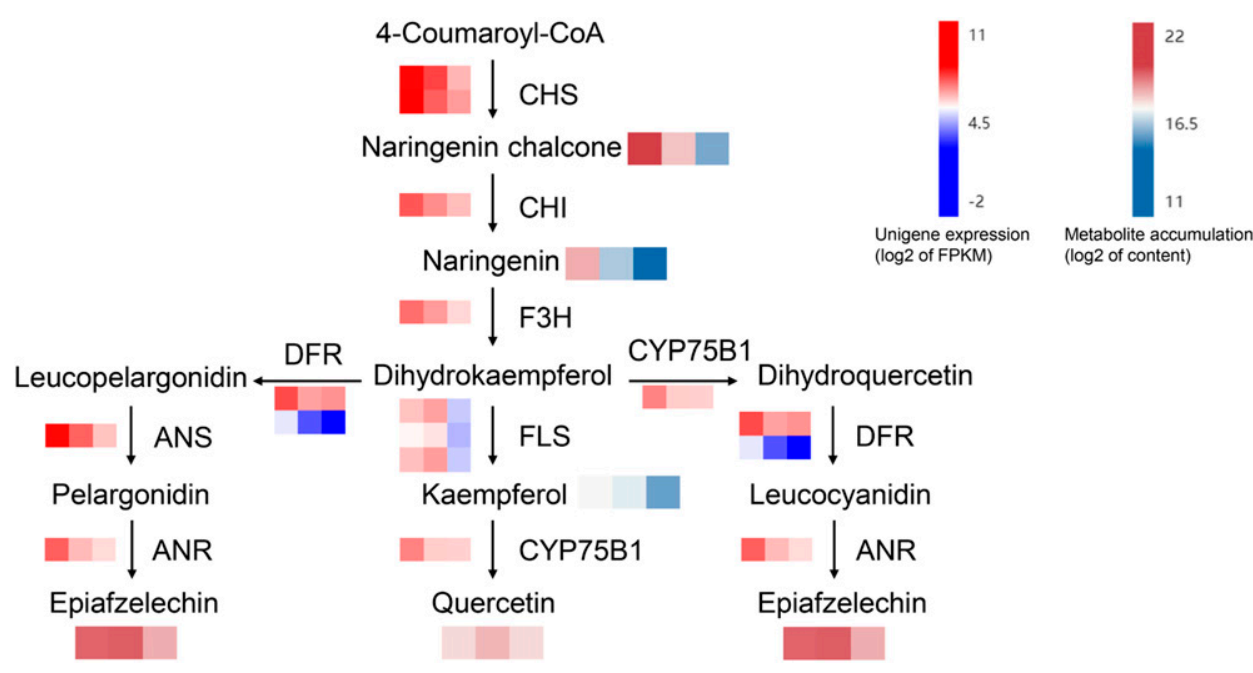

Fig. 8. Changes in gene expression and abundance of metabolite in the Lithocarpus polystachyus flavonoid biosynthesis pathway. From left to right, each box represents young, mature, and old leaves, respectively (CHS = chalcone synthase; $\mathrm{CHI}=$ chalcone flavanone isomerase; $\mathrm{F} 3 \mathrm{H}=$ flavanone 3-hydroxylase; FLS = flavonol synthase; ANR = anthocyanidin reductase; ANS = leucoanthocyanidin dioxygenase; DFR = flavanone 4reductase; CYP75B1 = flavonoid $3^{\prime}$-monooxygenase).

\section{Conclusions}

As the most commonly found metabolites in the leaves of $L$. polystachyus, flavonoids accumulate at the highest level during the early stage of development. Our study provides direct evidence of the correlation of gene expression and metabolites related to flavonoid biosynthesis, and it suggests the key role of the first few steps of flavonoid biosynthesis involving chalcone synthase and chalcone isomerase.

\section{Literature Cited}

Anders, S. and W. Huber. 2010. Differential expression analysis for sequence count data. Genome Biol. 11:R106, doi: 10.1186/gb-201011-10-r106.

Ashburner, M., C.A. Ball, J.A. Blake, D. Botstein, H. Butler, J.M. Cherry, A.P. Davis, K. Dolinski, S.S. Dwight, J.T. Eppig, et al. 2000. Gene Ontology: tool for the unification of biology. Nat. Genet. 25:25-29, doi: 10.1038/75556.

Benjamini, Y. and Y. Hochberg. 1995. Controlling the false discovery rate: A practical and powerful approach to multiple testing. J. R. Stat. Soc. B 57(1):289-300, doi: 10.1111/j.2517-6161.1995.tb02031.x.

Benson, D.A., I. Karsch-Mizrachi, D.J. Lipman, J. Ostell, and E.W. Sayers. 2010. GenBank. Nucleic Acids Res. 38:D46-D51, doi: 10.1093/nar/gkp1024.

Boeckmann, B., A. Bairoch, R. Apweiler, M.C. Blatter, A. Estreicher, E. Gasteiger, M.J. Martin, K. Michoud, C. O’Donovan, I. Phan, S. Pilbout, and M. Schneider. 2003. The SWISS-PROT protein knowledgebase and its supplement TrEMBL in 2003. Nucleic Acids Res. 31:365-370, doi: 10.1093/nar/gkg095.

Chen, N., J. Wang, Y. He, Y. Xu, Y. Zhang, Q. Gong, C. Yu, and J. Gao. 2020. Trilobatin protects against a $\beta_{25-35}$-induced hippocampal ht 22 cells apoptosis through mediating ros/p38/caspase 3-dependent pathway. Front. Pharmacol. 11:584, doi: 10.3389/fphar.2020.00584. Dong, H.-Q., M. Li, F. Zhu, F.-L. Liu, and J.-B. Huang. 2012. Inhibitory potential of trilobatin from Lithocarpus polystachyus Rehd against $\alpha$-glucosidase and $\alpha$-amylase linked to type 2 diabetes. Food Chem. 130:261-266, doi: 10.1016/j.foodchem.2011.07.030.

Escribano-Ferrer, E., J. Queralt Regue, X. Garcia-Sala, A. Boix Montanes, and R.M. Lamuela-Raventos. 2019. In vivo anti-inflammatory and antiallergic activity of pure naringenin, naringenin chalcone, and quercetin in mice. J. Nat. Prod. 82:177-182, doi: 10.1021/ acs.jnatprod.8b00366.

Finn, R.D., A. Bateman, J. Clements, P. Coggill, R.Y. Eberhardt, S.R. Eddy, A. Herger, K. Hetherington, L. Holm, J. Mistry, E.L.L. Sonnhammer, J. Tate, and M. Punta. 2014. Pfam: The protein families database. Nucleic Acids Res. 42 ( D 1): D $222-$ D 230 , doi: 10.1093/nar/gkt1223.

Gao, G., P. Chen, J. Chen, K. Chen, X. Wang, A.S. Abubakar, N. Liu, C. Yu, and A. Zhu. 2019. Genomic survey, transcriptome, and metabolome analysis of Apocynum venetum and Apocynum hendersonii to reveal major flavonoid biosynthesis pathways. Metabolites 9, doi: 10.3390/ metabo9120296.

Gao, J., N. Chen, N. Li, F. Xu, W. Wang, Y. Lei, J. Shi, and Q. Gong. 2020. Neuroprotective effects of trilobatin, a novel naturally occurring sirt3 agonist from Lithocarpus polystachyus Rehd., Mitigate cerebral ischemia/reperfusion injury: Involvement of TLR4/ NF- $\kappa \mathrm{B}$ and Nrf2/Keap-1 signaling. Antioxid. Redox Signal. 33:117-143, doi: 10.1089/ars.2019.7825.

Gaucher, M., T. Duge de Bernonville, D. Lohou, S. Guyot, T. Guillemette, M.N. Brisset, and J.F. Dat. 2013. Histolocalization and physico-chemical characterization of dihydrochalcones: Insight into the role of apple major flavonoids. Phytochemistry 90:78-89, doi: 10.1016/j.phytochem.2013.02.009.

Grabherr, M.G., B.J. Haas, M. Yassour, J.Z. Levin, D.A. Thompson, I. Amit, X. Adiconis, L. Fan, R. Raychowdhury, Q. Zeng, Z. Chen, E. Mauceli, N. Hacohen, A. Gnirke, N. Rhind, F. di Palma, B.W. Birren, C. Nusbaum, K. Lindblad-Toh, N. Friedman, and A. Regev. 2011. Full-length transcriptome assembly from RNA-Seq data without a reference genome. Nat. Biotechnol. 29:644-652, doi: 10.1038/ nbt. 1883.

Guo, C., H. Zhang, X. Guan, and Z. Zhou. 2019. The anti-aging potential of Neohesperidin and its synergistic effects with other citrus flavonoids in extending chronological lifespan of Saccharomyces cerevisiae BY4742. Molecules 24:4093, doi: 10.3390/molecules 24224093.

Guo, J., Y. Wu, G. Wang, T. Wang, and F. Cao. 2020. Integrated analysis of the transcriptome and metabolome in young and mature leaves of Ginkgo biloba L. Ind. Crops Prod. 143:111906, doi: 10.1016/j.indcrop.2019.111906.

Gutierrez, E., A. Garcia-Villaraco, J.A. Lucas, A. Gradillas, F.J. Gutierrez-Manero, and B. Ramos-Solano. 2017. Transcriptomics, targeted metabolomics and gene expression of blackberry leaves and fruits indicate flavonoid metabolic flux from leaf to red fruit. Front. Plant. Sci. 8:472, doi: 10.3389/fpls.2017.00472.

Hattori, M., N. Tanaka, M. Kanehisa, and S. Goto. 2010. SIMCOMP/ SUBCOMP: Chemical structure search servers for network analyses. Nucleic Acids Res. 38:W652-W656, doi: 10.1093/nar/ gkq367.

Hernández-Aquino, E. and P. Muriel. 2018. Beneficial effects of naringenin in liver diseases: Molecular mechanisms. World J. Gastroenterol. 24:1679-1707, doi: 10.3748/wjg.v24.i16.1679.

Huerta-Cepas, J., K. Forslund, L.P. Coelho, D. Szklarczyk, L.J. Jensen, C. Von Mering, and P. Bork. 2017. Fast genome-wide functional annotation through orthology assignment by eggNOG-mapper. Mol. Biol. Evol. 34(8):2115-2122, doi: 10.1093/molbev/msx148.

Hou, S.Z., S.J. Xu, D.X. Jiang, S.X. Chen, L.L. Wang, S. Huang, and X.P. Lai. 2012. Effect of the flavonoid fraction of Lithocarpus 
polystachyus Rehd. on spontaneously hypertensive and normotensive rats. J. Ethnopharmacol. 143:441-447, doi: 10.1016/ j.jep.2012.06.016.

Hou, S.Z., S.X. Chen, S. Huang, D.X. Jiang, C.J. Zhou, C.Q. Chen, Y.M. Liang, and X.P. Lai. 2011. The hypoglycemic activity of Lithocarpus polystachyus Rehd. leaves in the experimental hyperglycemic rats. J. Ethnopharmacol. 138:142-149, doi: 10.1016/ j.jep.2011.08.067.

Huang, H., Q. Yao, E. Xia, and L. Gao. 2018. Metabolomics and transcriptomics analyses reveal nitrogen influences on the accumulation of flavonoids and amino acids in young shoots of tea plant (Camellia sinensis L.) associated with tea flavor. J. Agr. Food Chem. 66:9828-9838, doi: 10.1021/acs.jafc.8b01995.

Kanehisa, M., S. Goto, S. Kawashima, Y. Okuno, and M. Hattori. 2004. The KEGG resource for deciphering the genome. Nucleic Acids Res. 32(90001):D277-D280, doi: 10.1093/nar/gkh063.

Koonin, E.V., N.D. Fedorova, J.D. Jackson, A.R. Jacobs, D.M. Krylov, K.S. Makarova, R. Mazumder, S.L. Mekhedov, A.N. Nikolskaya, B.S. Rao, I.B. Rogozin, S. Smironov, A.V. Sorokin, A.V. Sverdlov, S. Vasudevan, Y.I. Wolf, J.J. Yin, and D.A. Natale. 2004. A comprehensive evolutionary classification of proteins encoded in complete eukaryotic genomes. Genome Biol. 5(2):R7, doi: 10.1186/ gb-2004-5-2-r7.

Li, D., Z. Deng, B. Qin, X. Liu, and Z. Men. 2012. De novo assembly and characterization of bark transcriptome using Illumina sequencing and development of EST-SSR markers in rubber tree (Hevea brasiliensis Muell. Arg.). BMC Genomics 13:192, doi: 10.1186/ 1471-2164-13-192.

Li, S.H., X.J. Wu, J.P. Yu, S.Y. Li, and Y.G. Han. 2008. Study on extraction technics and content variety trends of total flavonoids in Lithocarpus polystachyus Rehd. Food Sci. 29:139-141, doi: 10.1016/S1872-5813(08)60024-9.

Li, Y., W. Guo, P. He, and L. Yu. 2019. The complete chloroplast genome of sweet tea (Lithocarpus polystachyus). Mitochondrial DNA B Resour. 4:2489-2490, doi: 10.1080/23802359.2019.1638841.

Leinonen, R., H. Sugawara, and M. Shumway. 2011. The Sequence Read Archive. Nucleic Acids Res. 39:D19-D21, doi: 10.1093/nar/ gkq1019.

Liu, Y., M. Li, J. Xu, X. Liu, S. Wang, and L. Shi. 2019. Physiological and metabolomics analyses of young and old leaves from wild and cultivated soybean seedlings under low-nitrogen conditions. BMC Plant Biol. 19:389, doi: 10.1186/s12870-019-2005-6.

Livak, K.J. and T.D. Schmittgen. 2001. Analysis of relative gene expression data using real-time quantitative PCR and the $2^{-\Delta \Delta C}{ }_{T}$ method. Methods 25:402-408, doi: 10.1006/meth.2001.

Loke, K.K., R. Rahnamaie-Tajadod, C.C. Yeoh, H.H. Goh, Z.A. Mohamed-Hussein, Z. Zainal, I. Ismail, and N. Mohd Noor. 2017. Transcriptome analysis of Polygonum minus reveals candidate genes involved in important secondary metabolic pathways of phenylpropanoids and flavonoids. PeerJ 5:e2938, doi: 10.7717/peerj.2938. Mariadoss, A.V.A., R. Vinyagam, V. Rajamanickam, V. Sankaran, S. Venkatesan, and E. David. 2019. Pharmacological aspects and potential use of phloretin: A systemic review. Mini Rev. Med. Chem. 19:1060-1067, doi: 10.2174/1389557519666190311154425.
R Core Team. 2014. R: A language and environment for statistical computing. R Foundation for Statistical Computing, Vienna, Austria. Rubel Mozumder, N.H.M., Y.-R. Lee, K.H. Hwang, M.-S. Lee, E.-H. Kim, and Y.-S. Hong. 2020. Characterization of tea leaf metabolites dependent on tea (Camellia sinensis) plant age through ${ }^{1} \mathrm{H}$ NMRbased metabolomics. Appl. Biol. Chem. 63: doi: 10.1186/s13765020-0492-7.

Shen, T., Y. Shang, Q. Wu, and H. Ren. 2020. The protective effect of trilobatin against isoflurane-induced neurotoxicity in mouse hippocampal neuronal HT22 cells involves the Nrf2/ARE pathway. Toxicology 442:152537, doi: 10.1016/j.tox.2020.152537.

Song, J., J. Huang, Z.D. Li, Y.H. Long, and Z.B. Xing. 2017. Analysis of transcriptomes and exploring flavonoid biosynthetic pathway genes in Lithocarpus polystachyus. China J. Chin. Mater. Med. 42:675-679.

Tajidin, N.E., K. Shaari, M. Maulidiani, N.S. Salleh, B.R. Ketaren, and M. Mohamad. 2019. Metabolite profiling of Andrographis paniculata (Burm. f.) Nees. young and mature leaves at different harvest ages using ${ }^{1} \mathrm{H}$ NMR-based metabolomics approach. Sci. Rep. 9:16766, doi: 10.1038/s41598-019-52905-z.

Tatusov, R.L., D.A. Natale, I.V. Garkavtsev, T.A. Tatusova, U.T. Shankavaram, B.S. Rao, B. Kiryutin, M.Y. Galperin, N.D. Fedorova, and E.V. Koonin. 2001. The COG database: New developments in phylogenetic classification of proteins from complete genomes. Nucleic Acids Res. 29:22-28, doi: 10.1093/nar/29.1.22.

Wang, F., W. Kong, G. Wong, L. Fu, R. Peng, Z. Li, and Q. Yao. 2016a. AtMYB12 regulates flavonoids accumulation and abiotic stress tolerance in transgenic Arabidopsis thaliana. Mol. Genet. Genomics 291:1545-1559, doi: 10.1007/s00438-016-1203-2.

Wang, J., Y. Huang, K. Li, Y. Chen, D. Vanegas, E.S. McLamore, and Y. Shen. 2016b. Leaf extract from Lithocarpus polystachyus Rehd. promote glycogen synthesis in T2DM mice. PLoS One 11:e 0166557, doi: 10.1371/journal.pone.0166557.

Yang, J., Y. Huang, Z. Yang, C. Zhou, and X. Hu. 2018. Identification and quantitative evaluation of major sweet ingredients in sweet tea (Lithocarpus polystachyus Rehd.) based upon location, harvesting time, leaf age. J. Chem. Soc. Pak. 40:158-164.

Yang, T., J. Li, H.X. Wang, and Y. Zeng. 2005. A geraniol-synthase gene from Cinnamomum tenuipilum. Phytochemistry 66:285-293, doi: 10.1016/j.phytochem.2004.12.004.

Zhang, S., Z.-F. Jiang, Q. Pan, C.-Y. Song, and W.-H. Zhang. 2016. Anti-cancer effect of naringenin chalcone is mediated via the induction of autophagy, apoptosis and activation of PI3K/Akt signaling pathway. Bangladesh J. Pharmacol. 11:684, doi: 10.3329/ bjp.v11i3.27518.

Zhang, Y., L. Lin, Y. Long, H. Guo, Z. Wang, M. Cui, J. Huang, and Z. Xing. 2019. Comprehensive transcriptome analysis revealed the effects of the light quality, light intensity, and photoperiod on phlorizin accumulation in Lithocarpus polystachyus Rehd. Forests 10:995, doi: 10.3390/f10110995.

Zhou, C.J., S. Huang, J.Q. Liu, S.Q. Qiu, F.Y. Xie, H.P. Song, Y.S. Li, S.Z. Hou, and X.P. Lai. 2013. Sweet tea leaves extract improves leptin resistance in diet-induced obese rats. J. Ethnopharmacol. 145:386-392, doi: 10.1016/j.jep.2012.09.05. 


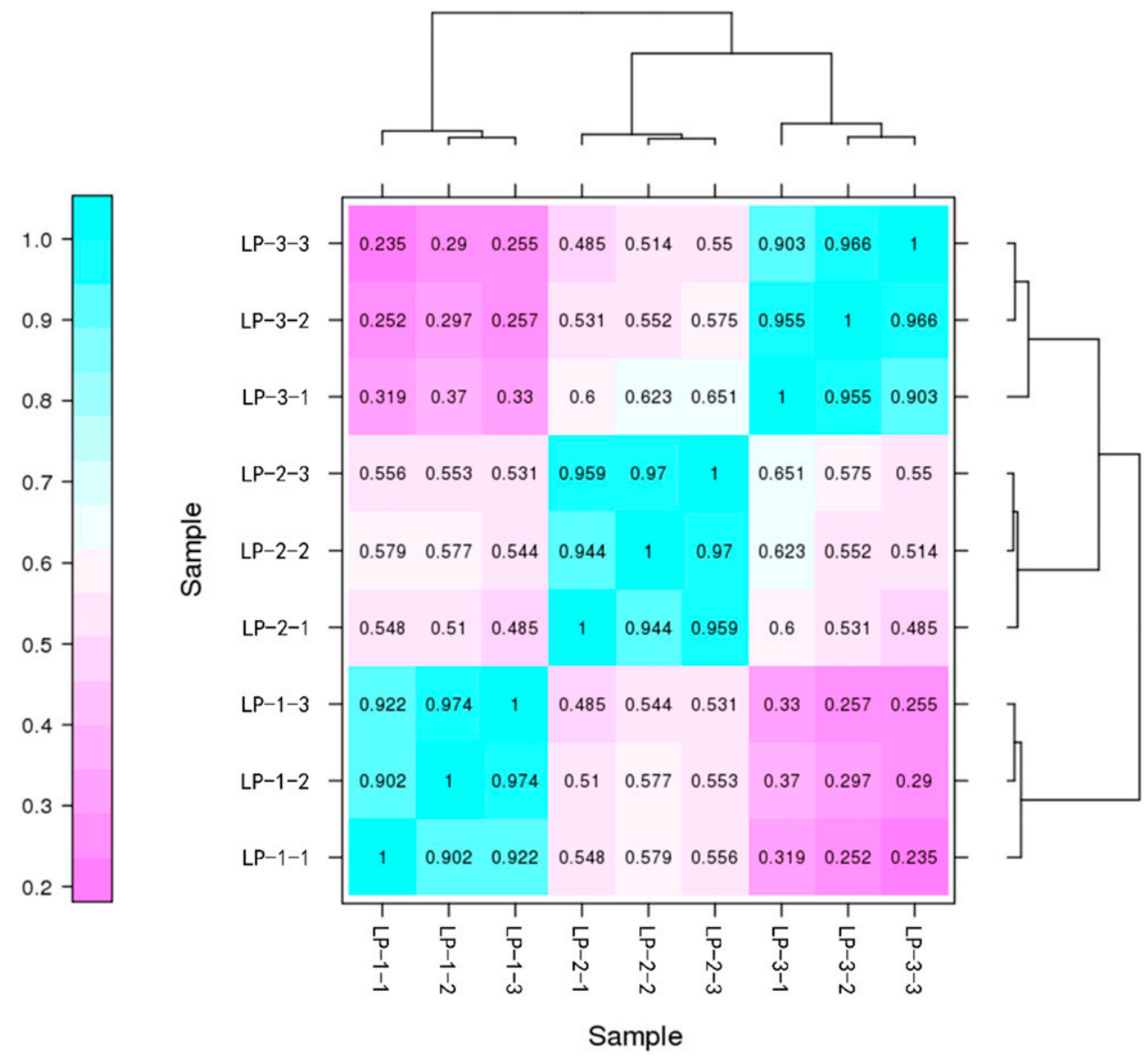

Supplemental Fig. 1. Correlation of RNA-seq data among nine samples of young (LP-1-1, LP-1-2, LP-1-3), mature (LP-2-1, LP-2-2, LP-2-3), and old (LP-3-1, LP3-2, LP-3-3) leaves of Lithocarpus polystachyus samples. Correlation coefficient values across samples were based on RNA-seq fragments per kilobase of transcript per million (FPKM). Cluster trees were based on distances of expressed genes. 
Supplemental Table 1. Primer sequences for quantitative reverse-transcription polymerase chain reaction (qRT-PCR) with Lithocarpus polystachyus leaves. The unigene ID, gene name, and forward and reverse primer sequences $\left(5^{\prime}\right.$ to $\left.3^{\prime}\right)$ are listed.

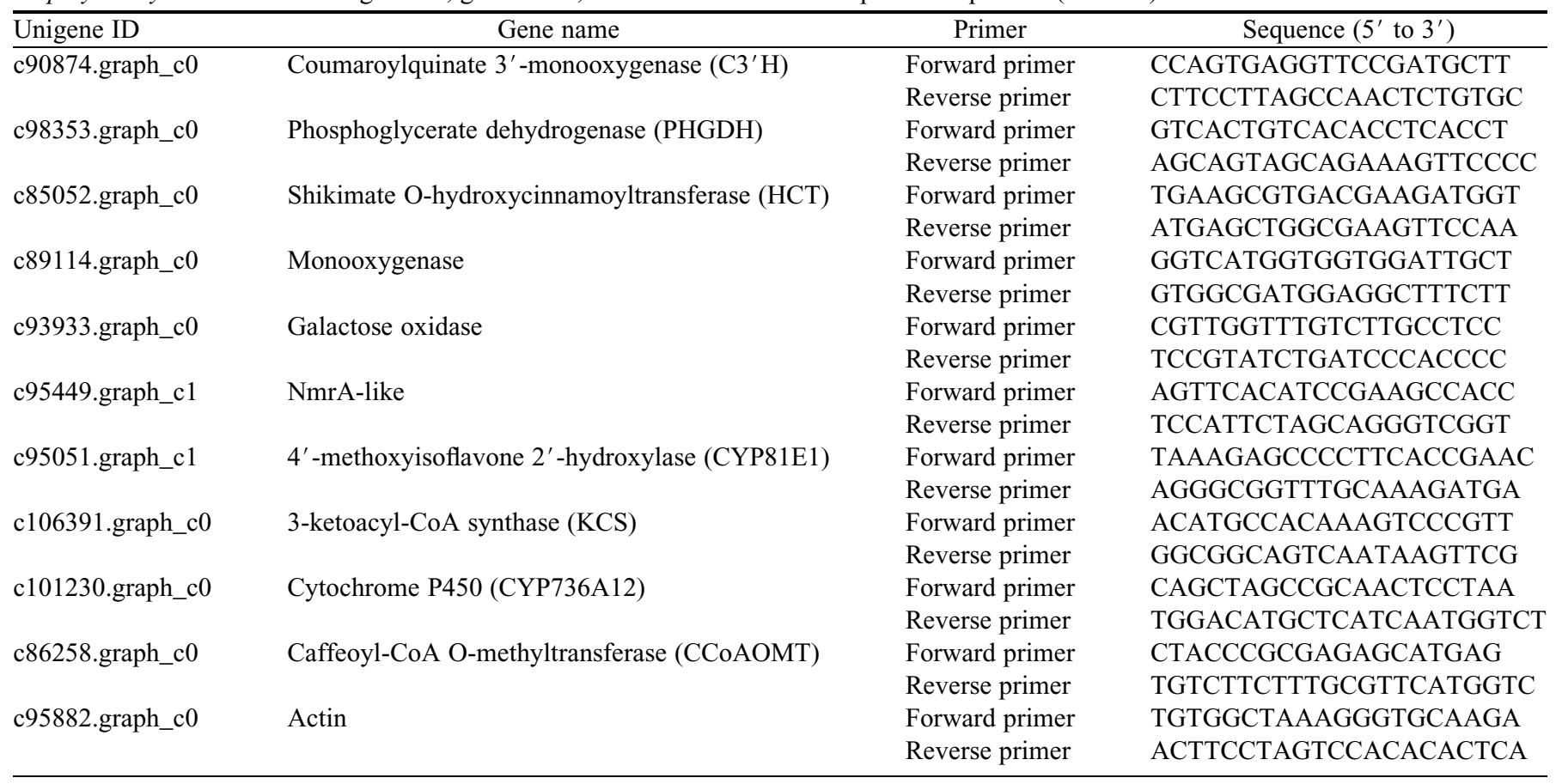


Supplemental Table 2. Gene Ontology (GO) classification of Lithocarpus polystachyus unigenes. GO_classify1 is the primary classification of the GO database. GO_classify2 is the secondary classification of the GO database.

\begin{tabular}{|c|c|c|}
\hline GO_classify1 & GO_classify2 & Unigenes (no.) \\
\hline Biological process & Metabolic process & 18,052 \\
\hline Biological process & Cellular process & 14,205 \\
\hline Biological process & Single organism process & 12,313 \\
\hline Biological process & Localization & 3,681 \\
\hline Biological process & Biological regulation & 3,560 \\
\hline Biological process & Cellular component organization or biogenesis & 2,368 \\
\hline Biological process & Multicellular organismal process & 1,471 \\
\hline Biological process & Signaling & 1,054 \\
\hline Biological process & Reproductive process & 833 \\
\hline Biological process & Multiorganism process & 766 \\
\hline Biological process & Growth & 298 \\
\hline Biological process & Reproduction & 276 \\
\hline Biological process & Locomotion & 10 \\
\hline Biological process & Cell killing & 1 \\
\hline Cellular component & Cell & 9,768 \\
\hline Cellular component & Cell part & 9,767 \\
\hline Cellular component & Organelle & 7,180 \\
\hline Cellular component & Membrane & 5,671 \\
\hline Cellular component & Organelle part & 3,224 \\
\hline Cellular component & Macromolecular complex & 3,158 \\
\hline Cellular component & Membrane part & 3,027 \\
\hline Cellular component & Membrane-enclosed lumen & 669 \\
\hline Cellular component & Extracellular region & 514 \\
\hline Cellular component & Extracellular matrix part & 1 \\
\hline Molecular function & Catalytic activity & 14,509 \\
\hline Molecular function & Binding & 12,497 \\
\hline Molecular function & Transporter activity & 1,731 \\
\hline Molecular function & Structural molecule activity & 1,110 \\
\hline Molecular function & Electron carrier activity & 496 \\
\hline Molecular function & Nucleic acid binding transcription factor activity & 418 \\
\hline Molecular function & Molecular transducer activity & 299 \\
\hline Molecular function & Antioxidant activity & 259 \\
\hline Molecular function & Enzyme regulator activity & 198 \\
\hline Molecular function & Receptor activity & 120 \\
\hline Molecular function & Protein-binding transcription factor activity & 46 \\
\hline Molecular function & Guanyl-nucleotide exchange factor activity & 42 \\
\hline Molecular function & Nutrient reservoir activity & 33 \\
\hline Molecular function & Metallochaperone activity & 5 \\
\hline Molecular function & Protein tag & 4 \\
\hline Molecular function & Channel regulator activity & 1 \\
\hline Molecular function & Translation regulator activity & 1 \\
\hline Total unigenes & & 25,099 \\
\hline
\end{tabular}

